\title{
Atypical and incomplete Kawasaki disease
}

\author{
Maria Cristina Maggio*, Giovanni Corsello \\ From 71st Congress of the Italian Society of Pediatrics. Joint National Meeting SIP, SIMGePeD, Study Group \\ on Pediatric Ultrasound, SUP Study Group on Hypertension \\ Rome, Italy. 4-6 June 2015
}

Incomplete Kawasaki disease (KD) occurs in patients with fever lasting five or more days and with two or three of the classical findings (exanthema, conjunctivitis, changes in the extremities, erythema of oral mucosa and lips, cervical lymphadenopathy) [1]. It is difficult to define its real incidence.

However the association between bilateral conjunctival bulbar injection and perineal erythema, with early desquamation in a patient with platelet count $>450.000$ after 7 days of fever is indicative of incomplete KD.

The definition "atypical KD" should be reserved for patients who have clinical manifestations such as renal impairment, unilateral peripheral facial nerve palsy, testicular swelling, pulmonary nodules and/or infiltrates, pleural effusions, diarrhea, vomiting and abdominal pain, acute surgical abdomen, hemophagocytic syndrome that generally are not seen in Kawasaki disease [1].

The "classical" clinical criteria have low sensitivity and specificity and therefore, other clinical and laboratory features may be helpful in establishing the diagnosis, especially for atypical or incomplete KD.

In the last years many studies highlighted clinical and/ or laboratory findings useful to obtain a precocious diagnosis in atypical and incomplete $\mathrm{KD}$, because coronary artery lesions (CAL) occur in $15-25 \%$ of untreated $\mathrm{KD}$ and approximately in $5 \%$ of $\mathrm{KD}$ who received intravenous immunoglobulin (IVIG) treatment before 10 days of fever $[2,3]$.

Relief of hyponatremia, hypoalbuminemia, increased D-Dimer are biochemical findings useful for diagnosis [4].

The association of more parameters, also considering AST, ALT, gamma-GT, leukocytes, percentage of neutrophils, platelet counts, CRP, ESR can be a help in the labyrinth of KD. In fact a precocious diagnosis and a timely

\footnotetext{
* Correspondence: sbenfratello@libero.it

University Department Pro.Sa.M.I. "G. D'Alessandro", University of Palermo,
} Palermo, 90134, Italy

treatment with IVIG significantly improve prognosis and reduce the risk of CAL.

However the doubt of an atypical KD requests electrocardiography and echocardiography, to exclude CAL, pericardial effusion, valvular insufficiency, arrhythmia, prolonged PR interval, nonspecific ST and T wave changes in patients with FUO. Although aneurysms are rare before day 10 of fever, perivascular brightness, coronary arteries ectasia in the acute stage, indicate coronary arteritis before the aneurysms develop.

Incomplete KD should be considered in all children with unexplained fever for $\geq 5$ days associated with 3 or less of the clinical criteria. incomplete $\mathrm{KD}$ is more common in young infants, who have a higher risk to develop CAL. Young infants may present with incomplete KD: echocardiography should be considered in infants younger than 6 months with fever for $\geq 7$ days, laboratory evidence of systemic inflammation, no other explanation of the disease.

\section{Published: 30 September 2015}

\section{References}

1. Newburger JW, Takahashi A, Gerber MA, Gewitz MH, Tani LY, Burns JC, et al: Diagnosis, treatment, and long-term management of Kawasaki disease: a statement for health professionals from the Committee on Rheumatic Fever, Endocarditis and Kawasaki Disease, Council on Cardiovascular Disease in the Young, American Heart Association. Circulation 2004, 110(17):2747-2771.

2. Kobayashi $T$, Inoue $Y$, Takeuchi $K$, Okada $Y$, Tamura $K$, Tomomasa $T$, et al: Prediction of intravenous immunoglobulin unresponsiveness in patients with Kawasaki disease. Circulation 2006, 113(22):2606-2612.

3. Do YS, Kim KW, Chun JK, Cha BH, Namgoong MK, Lee HY: Predicting factors for refractory kawasaki disease. Korean Circ J 2010, 40(5):239-242.

. Masuzawa Y, Mori M, Hara T, Inaba A, Oba MS, Yokota S: Elevated D-dimer level is a risk factor for coronary artery lesions accompanying intravenous immunoglobulin-unresponsive Kawasaki disease. Ther Apher Dial 2015, 19(2):171-177.

doi:10.1186/1824-7288-41-S2-A45

Cite this article as: Maggio and Corsello: Atypical and incomplete Kawasaki disease. Italian Journal of Pediatrics 2015 41(Suppl 2):A45. 